\title{
Momentum Spectra for Dynamically Assisted Schwinger Pair Production
}

\author{
M. Orthaber ${ }^{\mathrm{a}, \mathrm{b}}$, F. Hebenstreit ${ }^{\mathrm{a}}$, R. Alkofer ${ }^{\mathrm{a}}$ \\ ${ }^{a}$ Institut für Physik, Karl-Franzens-Universität Graz, A-8010 Graz, Austria \\ ${ }^{b}$ Materials Center Leoben Forschung GmbH., A-8700 Leoben, Austria
}

\begin{abstract}
Recently the dynamically assisted Schwinger mechanism, i.e., electron-positron pair production from vacuum by a combination -of laser pulses with different time scales has been proposed. The corresponding results, which suggest that the rate of produced pairs is significantly enhanced by dynamical effects, are verified. Employing the framework of quantum kinetic theory intrinsically 'enables us to additionally provide momentum space information on the generated positron spectrum.
\end{abstract}

'Keywords: Schwinger mechanism, Strong-field QED, Dynamically assisted Schwinger pair production

\section{Introduction}

The description of a charged particle within Quantum Electrodynamics (QED) necessitates to take the corresponding antiparticle into account. Historically, the first example has been the prediction of the positron, the antiparticle of the electron. One may exploit the similarity of quantum field theory to manybody physics by introducing the Dirac sea picture: The vacuum is modeled by a sea of fermions with all negative-energy levels filled. The antiparticle is then represented by a hole in this sea. This makes plain that an antiparticle-particle pair can be created as soon as enough energy is available to bridge the mass gap.

It became evident soon that one can create an antiparticleparticle pair either perturbatively from photons in high-energy reactions or non-perturbatively in the presence of very strong electric fields. The latter type has been called Schwinger mechanism [1] despite the fact that it has been discussed already many years before [2, 3]. This effect has attracted a lot of interest recently [4, 5, 6, 7, 8, 9, 10, 11, 12, 13] since laser facilities 'such as the Extreme Light Infrastructure (ELI) or the European XFEL might possibly reach the required field strengths.

Being aware of the possibilities of perturbative versus nonperturbative production mechanisms, the question arises how to -switch in a quasi-continuous way from one to the other. In this respect it turns out that time-dependent strong electric fields do this when varying the relevant frequency. Consequently, allowing for different and suitably chosen frequencies leads to a kind of dynamically assisted Schwinger mechanism [7].

The aim of the investigation reported in this letter is twofold: First, the mechanism as such is verified employing a quantum kinetic equation instead of the (semi-classical) world-line formalism. Second, and in view of the experiments even more important, momentum spectra of the produced pairs are predicted. Whilst the considered case is certainly not realistic in the sense of representing experimentally relevant superpositions of electric fields, it already demonstrates very nicely what kind of visible effects in the momentum spectra are to be expected in appropriately tuned upcoming experiments.
This letter is structured as follows: In Sect. 2 we set the theoretical basis for our investigations and introduce a simple model for dynamically assisted Schwinger mechanism 11 which is based on the proposal [7]. In Sect. 3]we discuss our numerical results on the momentum spectrum as well as on the particle density. We show that combining the mechanisms of perturbative and non-perturbative pair creation results in strong non-linear behavior which in turn leads to an enhancement in the particle yield.

\section{Pair Production in Multiple Time Scale Fields}

\subsection{Homogeneous Background Field Approximation}

With the advent of high-intensity laser facilities such as ELI or the European XFEL, we hope that it will become possible to create field strengths of the order of $\mathcal{E}_{c}=\mathrm{m}^{2} / e \sim 10^{16} \mathrm{~V} / \mathrm{cm}$ in the focus of colliding laser pulses. A laser should be described as an ensemble of photons in principle, showing both temporal and spatial variation. However, due to the fact that a complete description of this system is not yet feasible, we will make several reasonable simplifications in our calculations:

- We consider very high field strengths. Consequently, it is a reasonable assumption to treat the laser as classical background field. We thus neglect processes which could lead to a possible depletion of the laser due to electromagnetic cascades as indicated recently [15, 16, 17].

- The Schwinger mechanism is driven by the electric field. Having in mind a situation of colliding laser pulses with canceling magnetic fields, we will ignore the magnetic field completely; for studies including magnetic field effects see e.g. [18, 19].

\footnotetext{
${ }^{1}$ For a detailed and self-contained description, the interested reader may refer to [14].
} 
- The electric field is space- and time-dependent in general. Nevertheless, since the achievable spatial focusing scale is orders of magnitude larger than the Compton wavelength, we will ignore any spatial dependence. For studies on the spatial dependence of the Schwinger mechanism see e.g. [20, 21, 22, 23].

Consequently, we assume a spatially homogeneous electric field which is represented by a vector potential in temporal gauge $A_{\mu}(t)=(0, \mathbf{A}(t))$. The spatial part is chosen to point into the $\mathbf{e}_{3}$-direction: $\mathbf{A}(t)=(0,0, A(t))$, such that the magnetic field vanishes, $\mathbf{B}(t)=\nabla \times \mathbf{A}(t)=0$, and the electric field reads:

$$
\mathbf{E}(t)=-\frac{d \mathbf{A}(t)}{d t}=(0,0, E(t))
$$

\subsection{Quantum Kinetic Formalism}

We employ the quantum kinetic formalism in order to obtain not only production rates but also to gain information on the momentum spectrum of created particles. This additional information is highly relevant, particularly with regard to a possible future detection of the Schwinger mechanism. The spectral information is encoded in the distribution function $f(\mathbf{q}, t)$ : It has to be stressed that there is no clear interpretation of $f(\mathbf{q}, t)$ as long as the electric field is present. At finite times it cannot be considered as distribution function of real particles but only as a mixture of real and virtual excitations. Consequently, $f(\mathbf{q}, t)$ might be interpreted as the momentum distribution for real particles only at asymptotic times $t \rightarrow \pm \infty$, when the external field is switched off. This interpretation is also supported by S-matrix theory which clearly states that the identification of excitations of quantum fields with its corresponding particles is only possible at asymptotic but not at intermediate times. Alternative interpretations, which do not account for this peculiarity of $f(\mathbf{q}, t)$, lead to somewhat astonishing results [6, 24].

The equation of motion for $f(\mathbf{q}, t)$ can be derived from canonical quantization, quantizing the spinor field fully but considering the electromagnetic field as given background [25, 26, 27]. Alternatively, a derivation based on a Hartree approximation of the relativistic Wigner function for QED is possible [23, 28]. Due to the fact that we are mostly interested in the sub-critical field strength regime $\mathcal{E} \ll \mathcal{E}_{c}$ where the expected densities are rather low, we neglect collisions of created particles [19, 29]. Additionally, the self-consistent field current due to created particles, which leads to an internal electric field and the decrease of the background, should be taken into account in principle. Detailed analysis, however, showed that these contribution can be safely neglected in the sub-critical field strength regime $\mathcal{E} \ll \mathcal{E}_{c}$ as well [19, 30, 31]. With these simplifications, the quantum kinetic equation for $f(\mathbf{q}, t)$ reads:

$$
\dot{f}(\mathbf{q}, t)=W(\mathbf{q}, t) \int_{-\infty}^{t} d t^{\prime} W\left(\mathbf{q}, t^{\prime}\right)\left[1-f\left(\mathbf{q}, t^{\prime}\right)\right] \cos \left[2 \Theta\left(\mathbf{q} ; t, t^{\prime}\right)\right],
$$

where $f(\mathbf{q}, t)$ accounts for both spin directions due to the absence of magnetic fields. Here,

$$
W(\mathbf{q}, t)=\frac{e E(t) \epsilon_{\perp}}{\omega^{2}(\mathbf{q}, t)} \quad \text { and } \quad \Theta\left(\mathbf{q} ; t_{1}, t_{2}\right)=\int_{t_{1}}^{t_{2}} d t^{\prime} \omega\left(\mathbf{q}, t^{\prime}\right),
$$

with $\mathbf{q}=\left(\mathbf{q}_{\perp}, q_{3}\right)$ being the canonical momentum; $e$ denotes the electron charge; $\epsilon_{\perp}^{2}=m^{2}+\mathbf{q}_{\perp}^{2}$ is the transverse energy squared, whereas $\omega^{2}(\mathbf{q}, t)=\epsilon_{\perp}^{2}+p_{3}(t)^{2}$ is the total energy squared? Alternatively, this equation may be expressed as linear, first order, ordinary differential equation (ODE) system [30]:

$$
\begin{aligned}
\dot{f}(\mathbf{q}, t) & =W(\mathbf{q}, t) v(\mathbf{q}, t) \\
\dot{v}(\mathbf{q}, t) & =W(\mathbf{q}, t)[1-f(\mathbf{q}, t)]-2 \omega(\mathbf{q}, t) u(\mathbf{q}, t) \\
\dot{u}(\mathbf{q}, t) & =2 \omega(\mathbf{q}, t) v(\mathbf{q}, t)
\end{aligned}
$$

with initial conditions $f\left(\mathbf{q}, t_{i}\right)=v\left(\mathbf{q}, t_{i}\right)=u\left(\mathbf{q}, t_{i}\right)=0$ at $t_{i} \rightarrow$ $-\infty$. Both the integro-differential equation (2) and the ODE system (3) are valid for any time-dependent electric field.

In addition to the spectral information, the second important observable is given by the asymptotic particle number density $n\left[e^{+} e^{-}\right]$, which is calculated directly from $f(\mathbf{q}, t)$ :

$$
n\left[e^{+} e^{-}\right]=\int \frac{d^{3} q}{(2 \pi)^{3}} f(\mathbf{q}, \infty) .
$$

\subsection{Keldysh Parameter}

In order to create electron-positron pairs out of the vacuum, it is necessary to overcome the threshold energy of $2 m c^{2}$ from the filled Dirac sea to the unoccupied energy levels. Basically, there are two mechanism to achieve this:

- Schwinger pair creation [1, 3]: Virtual electron-positron fluctuations may gain the necessary energy $e \mathcal{E} L>2 m c^{2}$ by acceleration over a distance $L$ in a static or slowly varying electric field $\mathcal{E}$. This effect might be considered as tunneling effect and is as such exponentially suppressed.

- Dynamical pair creation [32, 33]: In a time-dependent electric field with characteristic frequency $\omega$, the threshold energy might be provided by a single photon $\hbar \omega>2 m c^{2}$. For $\hbar \omega<2 m c^{2}$, this effect might still occur by absorbing a multiple number of photons, but then becomes strongly suppressed as well.

An important parameter facilitating this characterization is the Keldysh parameter, first introduced in the context of atomic ionization [34] but later also taken over in the context of electronpositron pair creation from vacuum [32]:

$$
\gamma=\frac{\tau_{T}}{\tau}=\frac{m}{e \mathcal{E} \tau} .
$$

Here, $\tau_{T}=m / e \mathcal{E}$ is the characteristic time of a tunneling event. On the other hand, $\tau$ denotes the characteristic time scale of the applied electric field. The ability to tunnel is facilitated by the electric field $\mathcal{E}$ which tilts the potential around the electric field axis, as shown in Fig. 1

\footnotetext{
${ }^{2}$ The well known connection between canonical and kinetic momentum is $\mathbf{p}(t)=\mathbf{q}-e \mathbf{A}(t)$.
} 

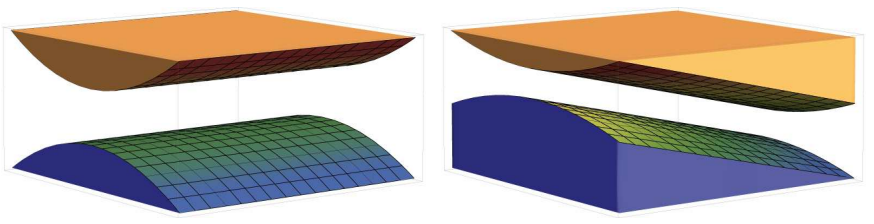

Figure 1: Left: Relativistic dispersion relation for vanishing electric field. Right: The electric field leads to a tilt in the relativistic dispersion relation such that tunneling out of the filled Dirac sea to the continuum is possible.

Consequently, the Keldysh parameter discriminates between two regimes:

- Adiabatic regime $\gamma \ll 1\left(\tau_{T} \ll \tau\right)$ : Tunneling through the barrier is possible since the electric field might be considered as static at any instant of time. In this regime, electron-positron pairs are created spontaneously by means of a non-perturbative tunneling process.

- Anti-adiabatic regime $\gamma \gg 1\left(\tau_{T} \gg \tau\right)$ : Tunneling through the barrier is not possible because the shape of the electric field changes much faster than the time needed to tunnel. In this perturbative regime, electron-positron pairs might only be created by absorption of the necessary threshold energy and not via a tunneling event.

\subsection{Electric Field with Two Time Scales}

Multi-photon pair creation has already been experimentally verified at the SLAC E-144 experiment [35], whereas the Schwinger mechanism has not been observed so far due to its strong exponential suppression of the order of $\exp \left(-\pi \mathcal{E}_{c} / \mathcal{E}\right)$. Hence, various mechanisms have been proposed in order to partly overcome this suppression of the Schwinger mechanism by taking advantage of pair creation in the multiphoton regime [7, 8, 12, 13].

The idea of the dynamically assisted Schwinger mechanism is rather simple [7]: Superimposing a strong but slowly varying electric field with a weak but rapidly varying electric field leads to a decrease of the tunneling barrier and, consequently, to a drastic enhancement of the electron-positron pair creation rate. As a very simple example we consider two Sauter-type electric fields 3

$$
E_{i}(t)=\mathcal{E}_{i} \operatorname{sech}^{2}\left(t / \tau_{i}\right)
$$

\footnotetext{
${ }^{3}$ Note, that due to the fact that the Dirac equation is exactly solvable in terms of hypergeometric functions in a single background field Eq. (7), we are able to find an analytic expression for the distribution function $f(\mathbf{q}, t)$ at both finite and asymptotic times [23]. For simplicity, we only give the result of the asymptotic momentum distribution here:

$$
f(\mathbf{q}, \infty)=\frac{2 \sinh \left(\pi\left[e \mathcal{E} \tau^{2}+\mu-v\right]\right) \sinh \left(\pi\left[e \mathcal{E} \tau^{2}-\mu+v\right]\right)}{\sinh (2 \pi \mu) \sinh (2 \pi v)},
$$

with

$$
\begin{aligned}
\mu & =\frac{\tau}{2} \sqrt{m^{2}+\mathbf{q}_{\perp}^{2}+\left(q_{3}-e \mathcal{E} \tau\right)} \\
v & =\frac{\tau}{2} \sqrt{m^{2}+\mathbf{q}_{\perp}^{2}+\left(q_{3}+e \mathcal{E} \tau\right)}
\end{aligned}
$$
}

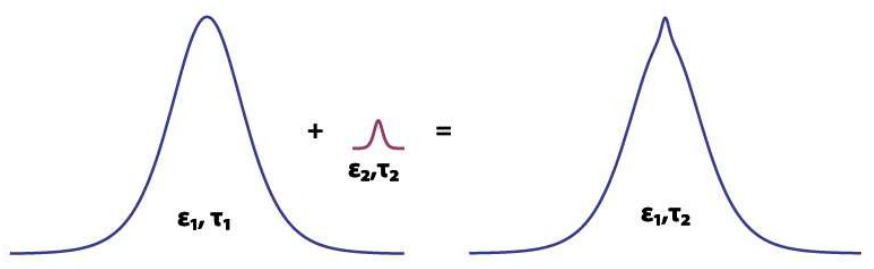

Figure 2: Superposition of two Sauter-type electric fields Eq. 8 for $t_{0}=0$. The relevant scales in this system are the larger field strength $\mathcal{E}_{1}$ and the shorter time scale $\tau_{2}$.

In our calculations we take $E_{1}(t)$ as the strong field, whereas $E_{2}(t)$ is considered as the weak field. Consequently, we have $\mathcal{E}_{2} \ll \mathcal{E}_{1}$ and $\tau_{2} \ll \tau_{1}$. In terms of the Keldysh parameter we have $\gamma_{1} \ll 1$ and $\gamma_{2} \gg 1$. We then consider the superposition of two such electric fields (cf. Fig. 2):

$$
E(t)=\mathcal{E}_{1} \operatorname{sech}^{2}\left(t / \tau_{1}\right)+\mathcal{E}_{2} \operatorname{sech}^{2}\left(\left(t-t_{0}\right) / \tau_{2}\right) .
$$

The parameter $t_{0}$ accounts for a possible shift of the individual pulse maxima with respect to each other.

It must be stressed that the relevant Keldysh parameter for this problem is neither $\gamma_{1}$ nor $\gamma_{2}$ but a combination of the dominant scales of the two different electric pulses [7]. These relevant scales are given by the larger field strength $\mathcal{E}_{1}$ and the shorter time scale $\tau_{2}$ :

$$
\gamma=\frac{m}{e \mathcal{E}_{1} \tau_{2}}
$$

This combination of different scales results in a subtle change in the pair production process by means of a non-trivial combination of Schwinger and dynamical pair creation. Consequently, the resulting distribution function $f(\mathbf{q}, t)$ will not be given by a naive addition of $f_{1}(\mathbf{q}, t)$ for the Schwinger mechanism and $f_{2}(\mathbf{q}, t)$ for dynamical pair creation, as we will see in the next section. In our calculations we consider a given strong electric field $E_{1}(t)$ in the adiabatic regime and a weak electric field $E_{2}(t)$ which can be varied by changing either the field strength $\mathcal{E}_{2}$ or the pulse length $\tau_{2}$.

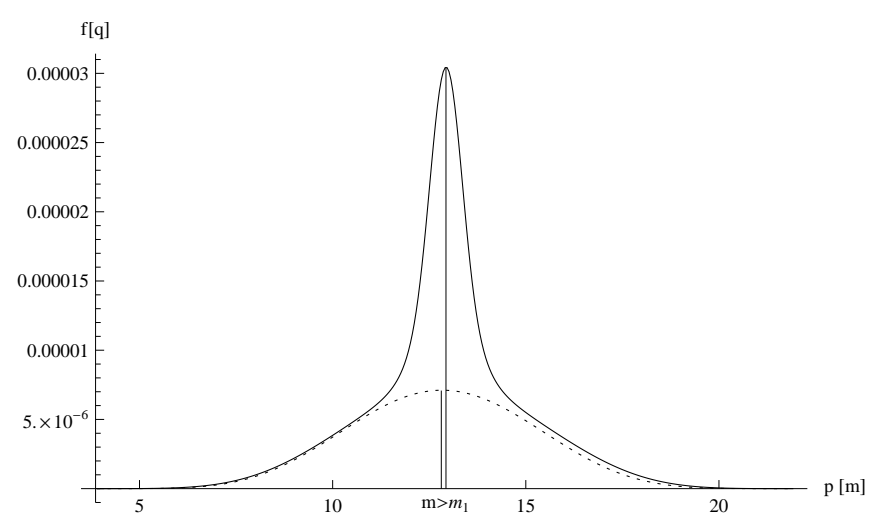

Figure 3: Comparison of the distribution function $f(\mathbf{q}, \infty)$ (solid line) with $f_{1}(\mathbf{q}, \infty)$ (dotted line). The positions of the maxima of the distribution functions are marked by $m$ and $m_{1}$, respectively. The parameters are given in the text. $f_{2}(\mathbf{q}, \infty)$ is not shown because it is orders of magnitude smaller. 


\section{Numerical Results}

We now turn to the numerical results for the dynamically assisted Schwinger mechanism in an electric field with multiple scales. In a first instance we focus on the asymptotic distribution function $f(\mathbf{q}, \infty)$ of created particles. Subsequently, we discuss the enhancement in the number density $n\left[e^{+} e^{-}\right]$of created particles in a specific parameter region due to the non-trivial combination of Schwinger and dynamical pair creation.

\subsection{Momentum Spectrum}

In Fig. 3 we consider the dynamically assisted Schwinger mechanism for a given setup, with the parameters for the strong pulse $\left\{\mathcal{E}_{1}=0.25 \mathcal{E}_{c}, \tau_{1}=10^{-4} \mathrm{eV}^{-1}\right\}$ and the weak pulse $\left\{\mathcal{E}_{2}=\right.$ $\left.0.025 \mathcal{E}_{c}, \tau_{2}=7 \cdot 10^{-6} \mathrm{eV}^{-1}\right\}$. For these parameters, $\gamma_{1} \approx 0.078$ is deep in the non-perturbative regime whereas $\gamma_{2} \approx 11.18$ is well in the multi-photon region. In this figure, we compare the momentum spectrum for a single Schwinger-like pulse with the momentum spectrum for a combined pulse Eq. (8) for $t_{0}=0$. We should first make two remarks:

- The parameter sets $\left\{\mathcal{E}_{1}, \tau_{1}\right\}$ and $\left\{\mathcal{E}_{2}, \tau_{2}\right\}$ are chosen such that the contribution due to Schwinger pair production $f_{1}(\mathbf{q}, \infty)$ is orders of magnitude larger than that due to dynamical pair production $f_{2}(\mathbf{q}, \infty)$. In order to observe the dynamically assisted Schwinger mechanism, it is of great importance to consider a parameter region which is in this sense dominated non-perturbatively (Schwinger-like).

- The distribution function for single pulses is easily found by the analytic formula Eq. (6). This formula also served as a numerics benchmark throughout this work.

We observe, that the momentum spectrum for the combined pulse $f(\mathbf{q}, \infty)$ is not given by naively adding $f_{1}(\mathbf{q}, \infty)$ and $f_{2}(\mathbf{q}, \infty)$ but shows a strong non-linear behavior. In fact, $f(\mathbf{q}, \infty)$ and $f_{1}(\mathbf{q}, \infty)$ nearly coincide for small kinetic momenta but differ for large kinetic momenta $f(\mathbf{q}, \infty)>f_{1}(\mathbf{q}, \infty)$. Additionally, the momentum spectrum is strongly enhanced around the maximum of $f_{1}(\mathbf{q}, \infty)$ (denoted as $m_{1}$ ) due to the influence of the short pulse $\left\{\mathcal{E}_{2}, \tau_{2}\right\}$. Moreover, the maximum of $f(\mathbf{q}, \infty)$ (denoted as $m$ ) is shifted a little towards higher momenta compared to the pure Schwinger mechanism $f_{1}(\mathbf{q}, \infty)$, i.e. $m>m_{1}$.

In order to better understand this behavior, let us consider the influence of different $t_{0}$ values on the pair creation process in Fig. 4 For $t_{0}<0$ (two upper figures), i.e. the maximum of the weak pulse occurs prior to the maximum of the strong pulse, we observe that the two curves coincide around $m_{1}$. Due to the fact that the weak pulse acts prior to the strong pulse, this field configuration behaves much like a single strong pulse. The only difference is that particles which are created by the weak pulse are accelerated by the subsequent strong pulse such that $m>m_{1}$. Oppositely for $t_{0}>0$ (two lower figures), i.e. the maximum of the weak pulse occurs after the maximum of the strong pulse. In this case, the particles which are created by the strong pulse get an extra kick by the subsequent weak pulse such that $f(\mathbf{q}, \infty)$ is shifted to larger momenta compared
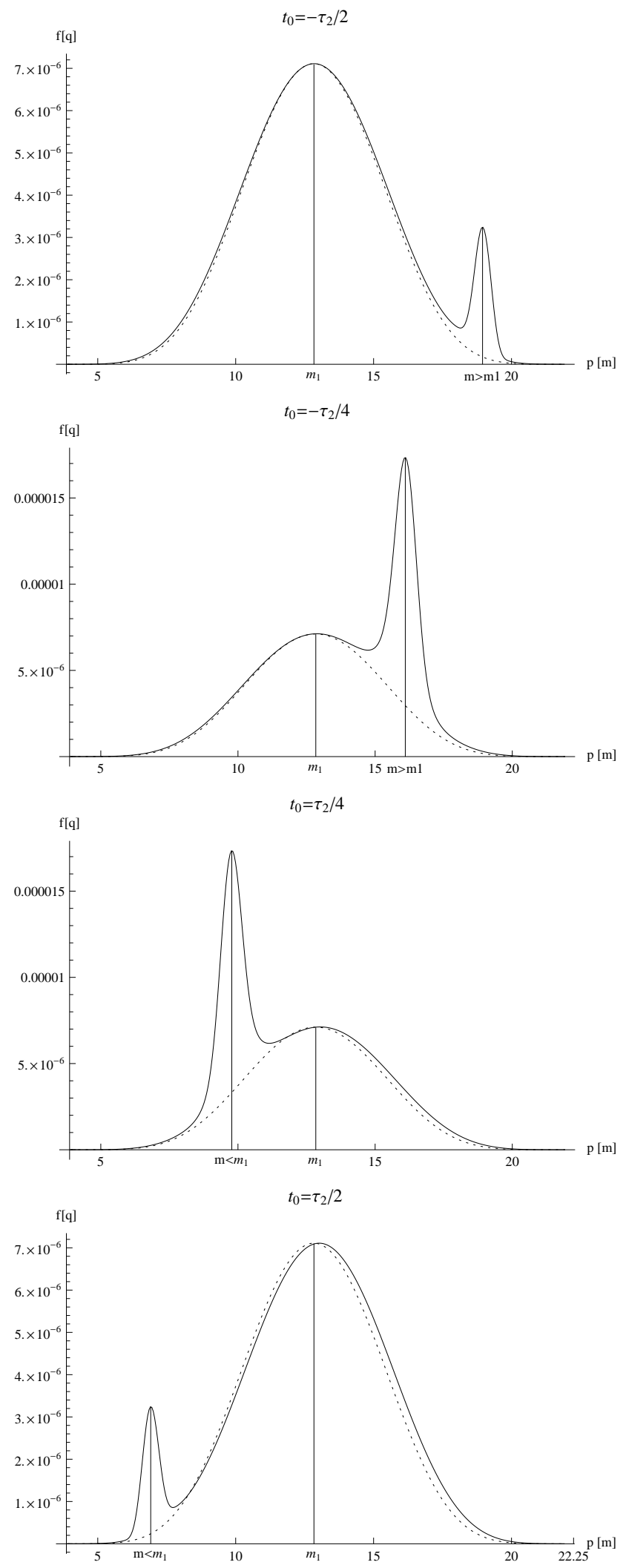

Figure 4: Comparison of the distribution function $f(\mathbf{q}, \infty)$ (solid line) with $f_{1}(\mathbf{q}, \infty)$ (dotted line). Depending on $t_{0}$, the maximum of the weak pulse occurs either prior or after the maximum of the strong pulse. The other parameters are chosen as in Fig. 3 
to $f_{1}(\mathbf{q}, \infty)$. Additionally, the pairs which are created by the weak pulse are accelerated less than in the previous case such that $m<m_{1}$.

For $t_{0}=0$ we observe a combination of those two effects (cf. Fig. 3): On the one hand we find that $f(\mathbf{q}, \infty)>f_{1}(\mathbf{q}, \infty)$ for large momenta, i.e. the particles get an extra kick by the weak pulse. On the other hand, we also find that $m>m_{1}$, i.e. the particles which are created by the weak pulse are still strongly accelerated by the strong pulse.

Due to the fact that we obtain the maximal enhancement at $t_{0}=0$, we consider this situation in the following. Before discussing the enhancement of the number density $n\left[e^{+} e^{-}\right]$in the next section, we want to investigate the momentum spectrum depending on the pulse length $\tau_{2}$ of the weak pulse in Fig. 5.

The weak pulse becomes shorter by decreasing the value of $\tau_{2}$, i.e., the energy of the corresponding quanta gets larger. Accordingly, it is getting easier to create an electron-positron pair via dynamical pair creation. As soon as the value of $\tau_{2} \lesssim 5 \cdot 10^{-6} \mathrm{eV}^{-1}$, we observe distinct momentum signatures around the maximum $m$ in tune with the inverse time scale $1 / \tau_{2}$. This might be interpreted as the onset of multi-photon domination, i.e., whilst the dynamically assisted Schwinger mechanism was still non-perturbatively dominated in Fig. 3, the effect becomes mostly perturbative in Fig. 5 .
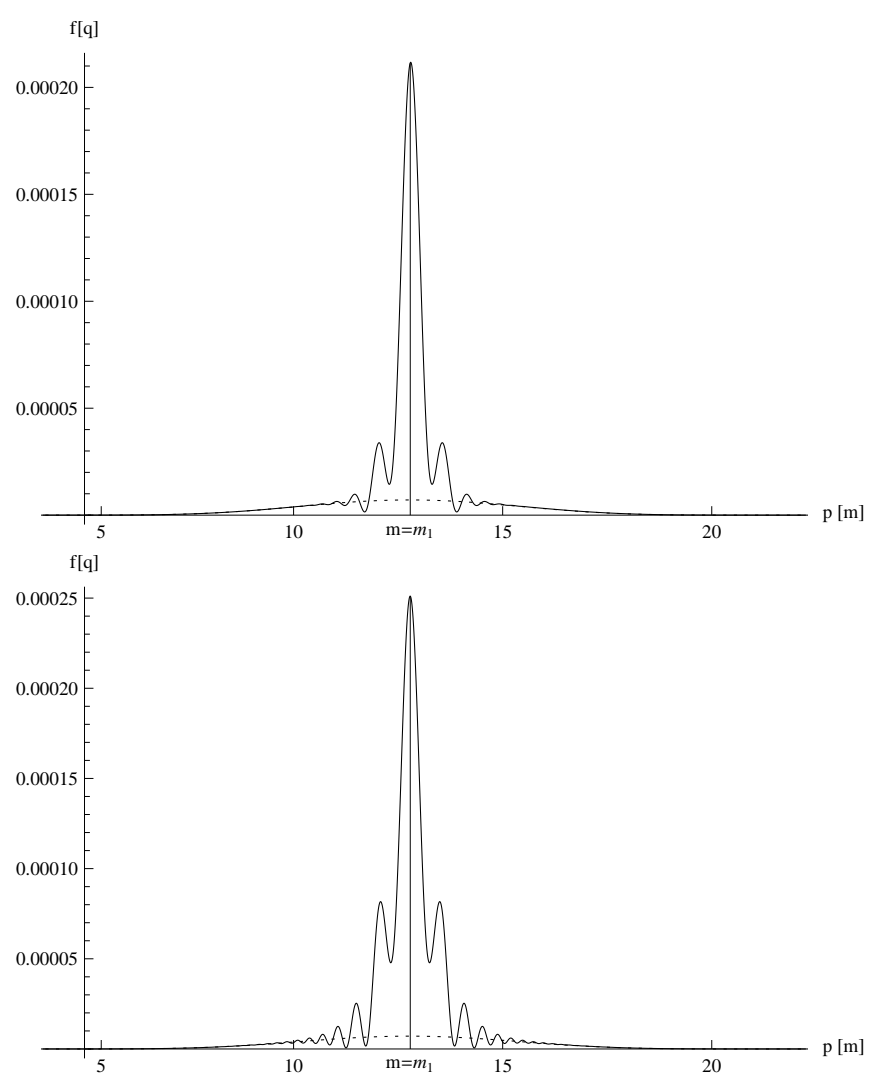

Figure 5: Comparison of the distribution function $f(\mathbf{q}, \infty)$ (solid line) with $f_{1}(\mathbf{q}, \infty)$ (dotted line) for different values of $\tau_{2}$. Top: $\tau_{2}=2 \cdot 10^{-6} \mathrm{eV}^{-1}$ Bottom: $\tau_{2}=10^{-6} \mathrm{eV}^{-1}$ The other parameters are chosen as in Fig. 3

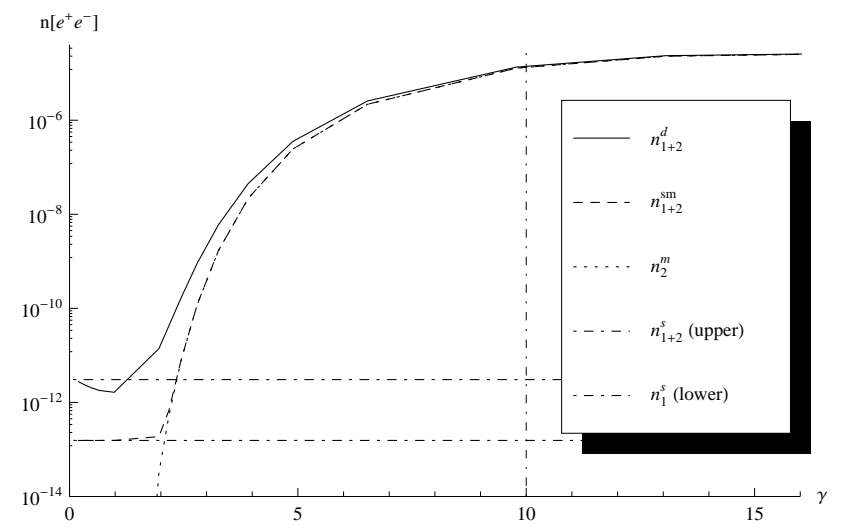

Figure 6: Comparison of the number density $n\left[e^{+} e^{-}\right]$according to the various effects. The parameters are given in the text.

\subsection{Number Density}

We now turn to the investigation of the number density $n\left[e^{+} e^{-}\right]$for the dynamically assisted Schwinger mechanism. In Fig. 6 we vary the pulse length $\tau_{2}$ of the weak pulse and, accordingly, the combined Keldysh parameter $\gamma$. The other parameters are chosen to be $\left\{\mathcal{E}_{1}=0.1 \mathcal{E}_{c}, \tau_{1}=2 \cdot 10^{-4} \mathrm{eV}^{-1}\right\}$ for the strong pulse (this corresponds to $\gamma_{1} \sim 0.097$ ) and $\left\{\mathcal{E}_{2}=0.01 \mathcal{E}_{c}, \tau_{2}\right.$ variable $\}$ for the weak pulse:

- The number density $n_{1}^{s}\left[e^{+} e^{-}\right]$of a single strong pulse $\left\{\mathcal{E}_{1}, \tau_{1}\right\}$ (pure Schwinger mechanism) is represented by the lower lying dashed-dotted line.

- The number density $n_{1+2}^{s}\left[e^{+} e^{-}\right]$of a single strong pulse $\left\{\mathcal{E}_{1}+\mathcal{E}_{2}, \tau_{1}\right\}$ (pure Schwinger mechanism) is represented by the higher lying dashed-dotted line.

- The number density $n_{2}^{m}\left[e^{+} e^{-}\right]$of a single weak pulse $\left\{\mathcal{E}_{2}, \tau_{2}\right\}$ (pure multi-photon effect) with $\tau_{2}$ being varied is represented by the dotted line.

- The number density $n_{1+2}^{s m}\left[e^{+} e^{-}\right]$which is obtained by naively adding the contributions of both the Schwinger and the multi-photon effect $n_{1}^{s}\left[e^{+} e^{-}\right]+n_{2}^{m}\left[e^{+} e^{-}\right]$is represented by the dashed line.

- The number density $n_{1+2}^{d}\left[e^{+} e^{-}\right]$which is obtained by our numerical calculation of the dynamically assisted Schwinger mechanism is represented by the solid line.

Additionally we have a dotted vertical line which corresponds to the parameter set at which the weak pulse has a time scale being equal to the Compton time of the electron, i.e., $\tau_{2}=1 / \mathrm{m}$. Note that these calculations have been done for $q_{\perp}=0$, i.e. ignoring the perpendicular momentum. Including the orthogonal momentum in the calculations is straightforward but not done for computational reasons. We observe the following behavior:

- For a very small Keldysh parameter $\gamma \ll 1$ such that $\tau_{2}=\tau_{1}$, we obtain the same result as the pure Schwinger mechanism with the parameters $\left\{\mathcal{E}_{1}+\mathcal{E}_{2}, \tau_{1}\right\}$. This is exactly what we expect since:

$$
E(t)=E_{1}(t)+E_{2}(t)=\left(\mathcal{E}_{1}+\mathcal{E}_{2}\right) \operatorname{sech}^{2}\left(t / \tau_{1}\right)
$$




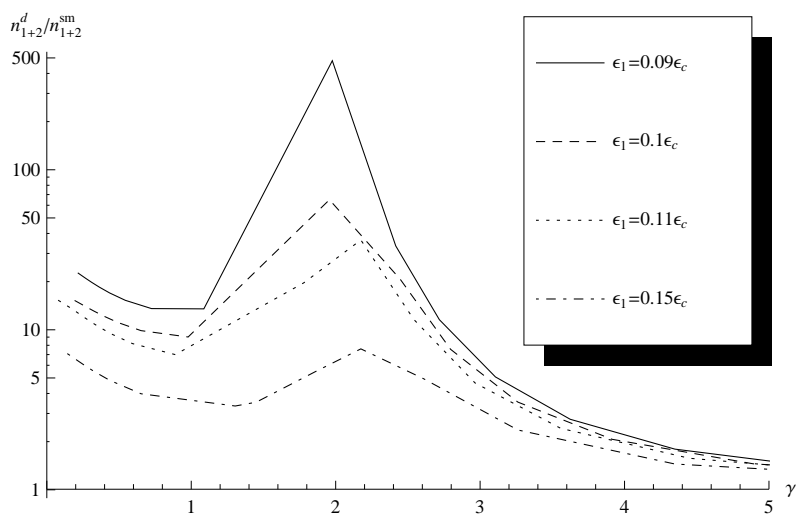

Figure 7: Relative Enhancement of the number density $n_{1+2}^{d}\left[e^{+} e^{-}\right] / n_{1+2}^{s m}\left[e^{+} e^{-}\right]$ due to the dynamically assisted Schwinger mechanism. The parameters are given in the text.

- For a very large Keldysh parameter $\gamma \gg 1$ the multiple time scale effect asymptotically coincides with the multiphoton result. This is reasonable because for very large $\gamma$ the multi-photon effect dominates the Schwinger mechanism by orders of magnitude.

- In between these two extreme cases we have the region which is of special interest to us. Here, we see clear signatures of the dynamically assisted Schwinger mechanism: For $1 \lesssim \gamma \lesssim 2$ we observe that $n_{1+2}^{s m}\left[e^{+} e^{-}\right]$, i.e. the naively added Schwinger and multi-photon effect, is well below $n_{1+2}^{d}\left[e^{+} e^{-}\right]$, i.e. the actual outcome of the dynamically assisted Schwinger mechanism.

Actually, the relative enhancement of the number density $n_{1+2}^{d}\left[e^{+} e^{-}\right] / n_{1+2}^{s m}\left[e^{+} e^{-}\right]$strongly depends on the various parameters, as shown in Fig. 7 To this end, we vary $\mathcal{E}_{1}$ but keep the ratio between the field strengths $\mathcal{E}_{2}=0.1 \mathcal{E}_{1}$ constant. Additionally, $\tau_{1}=2 \cdot 10^{-4} \mathrm{eV}^{-1}$ whereas $\tau_{2}$ is varied again.

The most important result is that in a region around $\gamma \sim 2$ the relative enhancement reaches a maximum with an enhancement factor of up to 500 for a given parameter set.

\section{Conclusions and Outlook}

We have presented a detailed calculation for an example of the dynamically assisted Schwinger mechanism which has been suggested recently [7, 8]. This mechanism, i.e., electronpositron pair production from vacuum by a combination of laser pulses with different time scales, suggests that the rate of produced pairs can be significantly enhanced by dynamical effects. First of all, we verified previous results, most prominently that one encounters a region around the Keldysh parameter $\gamma \sim 2$ where the relative enhancement reaches a maximum. Hereby, enhancement factors up to 500 have been obtained. Second, whereas the former investigations [7, 8] used the world-line instanton approach, the presented calculations are carried out within the framework of quantum kinetic theory. This method intrinsically enables us to additionally present valuable momentum space information of the generated positron spectrum.
On the basis of these results we conclude that the search for the dynamically assisted Schwinger mechanism could be facilitated by an ideal parameter choice in future experiments. It is obvious that the trivial combination of the two electric pulses considered here is neither optimal nor accomplishable experimentally. Therefore, a natural next step will be the investigation of more realistic electric pulses tuning the parameters for an increased pair production. Such pulse shaping in order to obtain an enhancement factor close to the greatest possible one even for more realistic electric field configurations is, although complicated, certainly feasible. Given the recent progress in theory and experiment a measurement of the Schwinger mechanism in this decade seems realistic.

\section{Acknowledgments}

We thank Gerald Dunne and Holger Gies for helpful discussions and a critical reading of the manuscript.

FH is supported by the DOC program of the Austrian Academy of Sciences and by the FWF doctoral program DK-W1203.

\section{References}

[1] J. S. Schwinger, Phys. Rev. 82 (1951) 664.

[2] F. Sauter, Z. Phys. 69 (1931) 742.

[3] W. Heisenberg and H. Euler, Z. Phys. 98 (1936) 714.

[4] R. Alkofer et al.,Phys. Rev. Lett. 87 (2001) 193902.

[5] A. Ringwald, Phys. Lett. B 510 (2001) 107.

[6] D. B. Blaschke et al.,Phys. Rev. Lett. 96, (2006) 140402.

[7] R. Schutzhold, H. Gies and G. Dunne, Phys. Rev. Lett. 101 (2008) 130404.

[8] G. V. Dunne, H. Gies and R. Schutzhold, Phys. Rev. D 80 (2009) 111301.

[9] F. Hebenstreit, R. Alkofer, G. V. Dunne and H. Gies, Phys. Rev. Lett. 102 (2009) 150404.

[10] F. Hebenstreit, R. Alkofer and H. Gies, Phys. Rev. D 78 (2008) 061701.

[11] M. Ruf et al.,Phys. Rev. Lett. 102 (2009) 080402.

[12] A. Di Piazza, E. Lotstedt, A. I. Milstein and C. H. Keitel, Phys. Rev. Lett. 103 (2009) 170403.

[13] S. S. Bulanov et al.,Phys. Rev. Lett. 104 (2010) 220404.

[14] M. Orthaber, Diploma thesis, University of Graz, 2010. |http://physik.uni-graz.at/ mao/|

[15] A. R. Bell and J. G. Kirk, Phys. Rev. Lett. 101 (2008) 200403.

[16] A. M. Fedotov, N. B. Narozhny, G. Mourou and G. Korn, Phys. Rev. Lett. 105 (2010) 080402.

[17] S. S. Bulanov et al. arXiv:1007.4306 [physics.plasm-ph].

[18] S. P. Kim and D. N. Page, Phys. Rev. D 73 (2006) 065020.

[19] N. Tanji, Annals Phys. 324 (2009) 1691.

[20] H. Gies and K. Klingmuller, Phys. Rev. D 72 (2005) 065001 |arXiv:hep-ph/0505099|.

[21] G. V. Dunne and Q. h. Wang, Phys. Rev. D 74 (2006) 065015.

[22] H. Kleinert, R. Ruffini and S. S. Xue, Phys. Rev. D 78 (2008) 025011.

[23] F. Hebenstreit, R. Alkofer and H. Gies, Phys. Rev. D 82 (2010) 105026.

[24] G. Gregori et al., High Energy Dens. Phys. 6 (2010) 166.

[25] Y. Kluger, E. Mottola and J. M. Eisenberg, Phys. Rev. D 58 (1998) 125015.

[26] S. A. Smolyansky et al. arXiv:hep-ph/9712377

[27] S. M. Schmidt et al.,Int. J. Mod. Phys. E 7 (1998) 709.

[28] I. Bialynicki-Birula, P. Gornicki and J. Rafelski, Phys. Rev. D 44 (1991) 1825.

[29] D. V. Vinnik et al.,Eur. Phys. J. C 22 (2001) 341.

[30] J. C. R. Bloch et al.,Phys. Rev. D 60 (1999) 116011.

[31] C. D. Roberts and S. M. Schmidt, Prog. Part. Nucl. Phys. 45 (2000) S1.

[32] E. Brezin and C. Itzykson, Phys. Rev. D 2 (1970) 1191. 
[33] V. S. Popov, Sov. Phys. JETP 34 (1972) 709; M. S. Marinov and V. S. Popov, Fortsch. Phys. 25 (1977) 373.

[34] L. V. Keldysh, Zh. Eksp. Teor. Fiz. 47 (1964) 1515 [Sov. Phys. JETP 20 (1965) 1018].

[35] C. Bamber et al., Phys. Rev. D 60 (1999) 092004. 